\title{
Development of a model protein for HIV Tat structural study and drug development
}

\author{
J Rose ${ }^{1}, \mathrm{Ji}^{2}$ \\ ${ }^{1}$ National Cancer Institute, Rockville, MD, ${ }^{2}$ National Cancer Institute, NIH, Frederick, MD \\ joshua.s.rose@gmail.com
}

Despite significant advances in antiretroviral treatment, HIV remains a global health issue with over 37.9 million individuals living with HIV in 2018. Treatment is highly effective at controlling the virus but has cytotoxic side effects and must be maintained for life or viral production will resume. Drugs that target additional HIV mechanisms can help overcome issues with resistant strains and provide new avenues for treatment. The $12-\mathrm{kDa}$ HIV Tat protein is a target for the development of new therapeutics. Tat is essential for viral replication to overcome the host RNA promoter proximal pausing defense mechanism. To continue paused transcription, Tat binds to a sequence of RNA in the 5' LTR of the HIV genome called TAR. The Tat:TAR complex recruits a host protein complex consisting of CDK9, AFF4, and CycT1 that rescues paused transcription. Despite extensive efforts to determine the molecular structure of the Tat:TAR complex, there is no structure of the entire C-terminal half of the 101 amino acid Tat protein, including the arginine rich motif (ARM) shown to be crucial for TAR binding. Efforts to target the Tat:TAR interaction through the development of small molecules and RNA mimetics have failed to produce viable drugs. Instead, we are targeting the CycT1:Tat interaction by using existing structural data to computationally design a small molecule inhibitor of the complex. Unfortunately, structural biology and structure-based drug discovery methods are hindered by the requirement that the protein be expressed in eukaryotic cells, generally insect cells, which reduces the ease of acquiring material. Here, we report the development of a linked chimera model protein consisting of CycT1 linked to the HIV Tat protein. The fusion of the two proteins increases their solubility, and by expressing the chimera protein with a cleavable maltose-binding protein tag the CycT1:Tat complex can be expressed and purified in large quantities from Escherichia coli cells. We determined the crystal structure of this CycT1:Tat protein bound to AFF4 and without RNA in space group P1 to $2.6 \AA$. As shown, the asymmetric unit contains four CycT1:Tat:AFF4 protomers. The structure matches previously published CycT1:Tat structures, showing a RMSD of $0.421 \AA$ A Using the CycT1-Tat chimera we are able to easily test numerous different lengths of the Tat protein for stability and RNA binding and subsequently determine the structure of Tat, containing the ARM, complexed with RNA. Additionally, our chimera protein will allow us to optimize our computationally designed inhibitor through structure-based drug design methods.

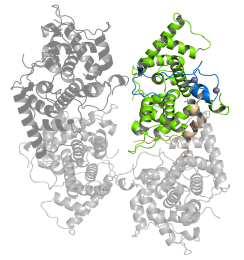

Figure 1

Acta Cryst. (2020). A76, a21 